\title{
Guest Editorial: Special Issue on Enterprise Systems
}

\author{
Albertas ČAPLINSKAS \\ Institute of Mathematics and Informatics \\ Akademijos 4, LT-01108 Vilnius, Lithuania \\ e-mail: alcapl@ktl.mii.lt
}

The term Enterprise System is interpreted and defined in many different ways. Up to date there is no single universally accepted definition. It is often thought of as a multilevel system composed of business, information processing and ICT levels. Other authors view it as "an integrated system, built on one database" (Strong and Volkoff, 2004) or as a single system that is central to the organization and ensures that information can be shared across all the functional levels and management hierarchies. Some others else (Carlock and Lane, 2006) argue that an Enterprise System should be viewed as a System of Systems or even as a complex system (Kuras, 2003), that is, an open dynamic system, for which any complete specification is not only impractical but also actually impossible. However, this Special Issue is not oriented to any precise understanding of an Enterprise System. It is viewed simply as an enterprise-wide system supporting strategic goals of the enterprise and meeting its operational needs. This Special Issue contains 6 papers on this topic.

J. Gøtze, P.E. Christiansen, R.K. Mortensen and S. Paszkowski discuss the issues of the development of Information Society. They analyze and assess the maturity level of the governmental enterprise architectures (EA) for 13 (mostly European) states. The analysis has highlighted that the major obstacles for the cross-national interoperability collaborations are the lack of top management support and political barriers.

E. Andonoff, W. Bouaziz, C. Hanachi and L. Bouzguenda also investigate the interoperability of Enterprise Systems. They propose an agent-based model for coordinating business processes involved in a dynamic inter-organizational workflow (IOW). This model allows bringing business process coordination issues back onto agent coordination issues and helps solving semantic conflicts using a shared ontology.

J. Pokorný discusses the problem of information integration in the Enterprise Systems. He investigates how XML technology contributes to finding a sufficiently powerful support for tools that enable such an integration. The paper summarizes the relevant parts of XML and demonstrates how XML databases can help to create more responsive Enterprise Information Integration architectures.

I. Magdalenic, D. Radosevic and Z. Skocir investigate the problem of data retrieval from heterogeneous data sources. They propose how, using ontologies, to dynamically generate web services for such data retrieval. 
A. Smaizys and O. Vasilecas discuss how to combine the business rules and enterprise resource planning systems. They compare three different approaches: parameterization of particular components of the ERP system; integration of business rules into the ERP system; and intelligent self adaptable ERP systems.

Finally, my own paper is devoted to the issues of Enterprise System requirement engineering and contributes to the further development of vision-driven requirements engineering techniques. It proposes a methodical framework that defines a complete scheme to organize the business level, user level, information system level, information system's subsystem level, and software level requirements and allows us to flowdown the requirements from business to the software level preserving their business-orientation.

I wish to express my appreciation to the authors, reviewers, and all the people involved in the preparation of this Special Issue. I believe that each of the papers contributes to the field of Enterprise Systems and I hope that the ideas proposed will be of interest to the readers of INFORMATICA.

\section{References}

Carlock, P.G., Lane, J.A. (2006). System of systems enterprise systems engineering, the enterprise architecture management framework, and system of systems cost estimation. In: Proc. of the 21st International Forum on COCOMO and System/Software Cost Modeling.

Kuras, M.L. (2003). Enterprises as complex systems. The Edge (Mitre Technology Newsletter), 7(2).

Strong, D.M., Volkoff, O. (2004). A roadmap for enterprise system implementation. Computer, 37(6), 22-29. 